\title{
Development and Evaluation of a Novel Delivery System Containing Phytophospholipid Complex for Skin Aging
}

\author{
Monica Damle ${ }^{1,3}$ (D) and Rashmi Mallya ${ }^{2}$
}

Received 6 May 2015; accepted 3 August 2015; published online 20 August 2015

\begin{abstract}
Citrus auranticum and Glycyrrhiza glabra are rich in anti-oxidant polyphenols helpful in prevention of skin aging. Polyphenols have high polarity and lower skin penetration resulting in lower cutaneous delivery. The present work is attempted to develop a novel polyherbal phospholipid complex cream to improve cutaneous delivery of polyphenols for sustained anti-oxidant action. Phytochemical and in vitro anti-oxidant evaluation was done on methanolic extracts of orange peel and liquorice powder. Total phenolic content, total flavonoid content, and anti-oxidant assays were done on different ratios of orange peel and liquorice extract. Ratio 1:2 gave highest total phenolic content (TPC) $(530.00 \pm 1.56 \mathrm{mg}$ gallic acid equivalent (GAE) $\mathrm{g}^{-1}$ extract), total flavonoid content (TFC) $(246.25 \pm 1.03 \mathrm{mg}$ rutin equivalent (RUE) ${ }^{-1}$ extract), 2,2-diphenyl-1-picrylhydrazyl (DPPH) scavenging activity (87.99 $\pm 0.64 \%$ ), and $\mathrm{H}_{2} \mathrm{O}_{2}$ scavenging activity $(72.47 \pm 0.86 \%)$ and hence was used for formulation. Solvent evaporation method using methanol with 1:1 extract to phospholipid ratio was found to have entrapment efficiency of $93.22 \pm 0.26 \%$. Evaluation parameters like scanning electron microscopy (SEM), Fourier transform infrared spectrophotometry (FT-IR), and differential scanning calorimetry (DSC) confirmed formation of complex. The complex was formulated as oil-in-water cream and evaluated for various parameters. The optimized cream containing $1 \%$ complex was non-irritant and was found to be stable for 3 -month period under conditions of stability study. Ex vivo diffusion studies showed that extract phospholipid complex cream had better retention of polyphenols in the skin when compared to conventional extract cream giving prolonged and stronger topical action. The cream had an anti-elastase activity of $28.02 \pm 0.95 \%$ at concentration of $3000 \mu \mathrm{g} \mathrm{ml}^{-1}(w / v)$. Thus, the developed safe and stable polyherbal phytophospholipid complex cream exhibited good potential as anti-aging cosmeceutical.
\end{abstract}

KEY WORDS: anti-aging; anti-oxidant; liquorice; orange peel; phytophospholipid complex.

\section{INTRODUCTION}

Skin, the most visible organ, performs crucial functions such as modulating body temperature and perceiving pressure, temperature, and pain and acts as fundamental barrier against pollution and environmental insults making aging very evident $(1,2)$. Aging manifests itself with thinning, sagging, appearance of age spots, and dryness of skin. Thus, with an increase in urge of staying or at least looking young, anti-aging products have towering demand.

Free radicals are highly reactive oxygen molecules that form cross linkages with collagen molecules causing the skin elasticity to lose tone, thus initiating aging process (1). Antioxidants prevent or slow down oxidative damage caused by free radicals (3). Anti-oxidant activity of herbal extracts is

\footnotetext{
${ }^{1}$ Department of Quality Assurance, Dr. Bhanuben Nanavati College of Pharmacy, Mumbai, India.

${ }^{2}$ Department of Pharmacognosy, Dr. Bhanuben Nanavati College of Pharmacy, Mumbai, India.

${ }^{3}$ To whom correspondence should be addressed. (e-mail: monicadamle6@gmail.com)
}

extremely useful in cosmetic formulations. This activity of herbs is mainly of due to the redox properties of phenolic compounds allowing them to act as reducing agents, hydrogen donors, and singlet oxygen quenchers (4). Many herbs, fruits, vegetables, and whole grains contain anti-oxidant polyphenols which scavenge and eliminate free radicals. Natural skin care products are hypoallergenic in nature and are absorbed quickly by the superficial layers of the skin (5). So, plants containing anti-oxidants can be used as anti-aging agents.

Orange peel (Citrus auranticum, Rutaceae) extract is a very effective anti-oxidant, anti-acne, and skin-whitening agent. The citrus peel and seeds are very rich in phenolic compounds like phenolic acids and flavonoids. The peels are richer in flavonoids compared to seeds (6). Liquorice (Glycyrrhiza glabra, Fabaceae) contains phenolic compounds (flavonoids) (7). The ethanol extract of liquorice has good anti-oxidant activity due to significant ROS scavenging, hydrogen-donating, and reducing abilities of phenolic components present in high amount (8). It improves viscoelastic and hydration properties of the skin (9).

Polyherbal formulations give high therapeutic effect due to the synergistic effect of chemical constituents present in the herbs (10). Orange peel extract and liquorice extract have 
been used individually for their potential cosmetic benefit. So, an attempt was made to study the combined effects of extracts on skin aging.

Stratum corneum of the skin is an excellent barrier to external application. In order to increase the skin permeation of the active ingredients, it is essential to use penetration enhancers or suitable vehicles. Phospholipids are employed in solubilized form as penetration enhancers $(11,12)$. The key advantage of phospholipids is a lower skin irritation tendency, when compared to typical penetration enhancers $(13,14)$.

Phytosomes $^{\circledR}$ is a patented technology developed and commercialized by Indena to incorporate standardized plant extracts or water-soluble constituents into phospholipids such as phosphatidylcholine (PC) derived from soy bean to produce lipid-compatible molecular complexes, called as Phytosomes ${ }^{\circledR}$. Phytophospholipid complexes (complexes of phytoconstituents and phospholipids) are useful in improving skin absorption and bioavailability of active ingredients. Phytophospholipid complexes give superior biological activity compared to an equal amount of the active ingredient or extract, orally and topically (1).

Thus, this study is focused on incorporation of phytophospholipid complex into a suitable topical dosage form for delivery to the skin ensuring increased cutaneous absorption and retention of polyphenols in skin for longer and stronger topical action against skin aging.

\section{MATERIALS AND METHODS}

\section{Materials}

C. auranticum and G. glabra powders used in this study were procured from local market in Mumbai in July 2013 and were authenticated by Dr. Bindu Gopalkrishnan at Botany Department, Mithibai College, Mumbai, India. LECIVA-S70 was a gift sample from VAV Life Sciences Pvt. Ltd., India. Gallic acid was procured from SD Fine-Chem Ltd., India. Rutin and ascorbic acid were procured from Loba Chemie, India. All other reagents and chemicals used in the study were of A.R. grade. Animals, albino Wistar rats (Rattus norvegicus) (180-220 g) of either sex, were obtained from Haffkine Institute, Mumbai, India. The experimental protocol was approved by the Institutional Animal Ethical Committee of Dr. Bhanuben Nanavati College of Pharmacy, Mumbai, India (CPCSEA/IAEC/BNCP/P-11/2014). Animal experiments were conducted as per the institute's guidelines of the animal ethics committee.

\section{Methods}

\section{Preparation of Crude Extract}

Methanolic extract of crude powders was obtained after Soxhlet extraction. Resulting extracts were concentrated using distillation apparatus and evaporated in a porcelain dish on electric water bath at $40^{\circ} \mathrm{C}$ to remove solvent.

\section{Total Phenolic Content(15)}

Total phenolic content was determined with the FolinCiocalteu reagent according to the procedure described by
Singleton and Rossi with slight modification. Gallic acid was used as a standard with concentrations ranging from 20 to $100 \mathrm{ppm}$. The solvent used was water. Briefly, $0.50 \mathrm{ml}$ of the sample (extract) and standard were reacted with $2.5 \mathrm{ml}$ of Folin-Ciocalteu reagent (1:10 diluted with water), and then 2 -ml saturated sodium carbonate solution $(7.5 \% \mathrm{w} / \mathrm{v})$ was added into the reaction mixture. The absorbance readings were taken using UV-vis spectrophotometer at $765 \mathrm{~nm}$ after incubation at room temperature for $15 \mathrm{~min}$. The experiment was performed in triplicate. The results were expressed as milligram of gallic acid equivalent per gram extract weight (mg GAE $\mathrm{g}^{-1}$ extract weight).

\section{Total Flavonoid Content(16)}

The total flavonoid content was done according to Marinova et al. One milliliter of the extracts and standards, respectively, were reacted with $3.4 \mathrm{ml}$ of methanol $(30 \%)$, $0.15 \mathrm{ml}(0.5 \mathrm{M})$ sodium nitrite $\left(\mathrm{NaNO}_{2}\right), 0.15 \mathrm{ml}(0.3 \mathrm{M})$ aluminum chloride $\left(\mathrm{AlCl}_{3}\right)$, and $1 \mathrm{ml}(1 \mathrm{M})$ sodium hydroxide $(\mathrm{NaOH})$. Rutin was used as a standard with concentrations ranging from 100 to $500 \mathrm{ppm}$. The experiment was performed in triplicate. The mixture was measured at $506 \mathrm{~nm}$ and expressed as milligram rutin equivalent per gram extract weight (mg RUE g ${ }^{-1}$ extract weight).

\section{Assessment of Anti-Oxidant Activity}

2,2-Diphenyl-1-Picrylhydrazyl Scavenging Activity (17). To $2 \mathrm{ml}$ of various test samples (extract solution 10 $60 \mathrm{ppm}$ ), $2 \mathrm{ml}$ solution of 2,2-diphenyl-1-picrylhydrazyl (DPPH) $0.1 \mathrm{mM}$ was added separately. The reaction mixture was shaken and incubated in the dark for $30 \mathrm{~min}$, at room temperature, and the absorbance was recorded at $517 \mathrm{~nm}$ against blank. Controls containing methanol instead of the anti-oxidant solution and blanks containing methanol instead of DPPH solution were also made. Ascorbic acid was used as the standard. The experiment was performed in triplicate. The inhibition of the DPPH radical by the samples was calculated with reference to control absorbance. The percentage of DPPH radical scavenging activity was plotted against the sample concentration. Scavenging activity was expressed as the inhibition percentage calculated using the following formula,

$$
\% \text { Inhibition }=\frac{\text { CONTROL Abs.-SAMPLE Abs. }}{\text { CONTROL Abs. }} \times 100
$$

Hydrogen Peroxide Scavenging Activity (18). The ability of the extracts to scavenge hydrogen peroxide $\left(\mathrm{H}_{2} \mathrm{O}_{2}\right)$ was determined according to the method of Ruch et al. with slight modifications. A solution of hydrogen peroxide $(43 \mathrm{mM})$ was prepared in phosphate buffer $\mathrm{pH}$ 7.4. Mixed were 0.1-ml (10$60 \mathrm{ppm}$ ) extract solution, $3.4 \mathrm{ml} 0.1 \mathrm{M}$ phosphate buffer $(\mathrm{pH}$ 7.4), and $0.6 \mathrm{ml} 43 \mathrm{mM} \mathrm{H}_{2} \mathrm{O}_{2}$, and the absorbance (Abs) of the mixture was measured at $230 \mathrm{~nm}$ against a blank solution containing phosphate buffer without hydrogen peroxide. Control solutions were prepared for each sample concentration by replacing the sample/standard with phosphate buffer. Ascorbic acid was used as the standard. The experiment was 
performed in triplicate. Hydrogen peroxide scavenging activity was expressed as the inhibition percentage calculated using the following formula,

$\%$ Inhibition $=\frac{\text { CONTROL Abs.-SAMPLE Abs. }}{\text { CONTROL Abs. }} \times 100$

\section{Correlation Studies}

Total phenolic content, total flavonoid content, and antioxidant assays were done on both the extracts and also on different ratios of orange peel and liquorice extract $(1: 1,1: 2$, $2: 3,3: 2,2: 1)$. Experimental results were further analyzed for Pearson correlation coefficient between total phenolic content (TPC), total flavonoid content (TFC), and different anti-oxidant assays and tested for significance by correlation bivariate analysis $(P<0.05)$. IBM SPSS ver. 21.0 and Microsoft Excel 2007 were used for the statistical and graphical evaluations.

\section{Combination Studies}

Different ratios of orange peel and liquorice extract (1:1, $1: 2,2: 3,3: 2,2: 1)$ were used for determination of TPC, TFC, and anti-oxidant assays. The ratio that gave the highest polyphenolic content and anti-oxidant effect was selected for the formulation.

\section{Phytophospholipid Complex Preparation}

Three different preparation techniques were tried in this study for complex formation, namely, film formation, saltingout, and solvent evaporation method.

\section{Refluxing with Methanol (Solvent Evaporation Method) (19)}

Specific amounts of extracts $(0.33 \mathrm{~g}$ orange peel extract and $0.66 \mathrm{~g}$ liquorice extract) and phospholipid (1 g) were refluxed at $50^{\circ} \mathrm{C}$ for $3 \mathrm{~h}$ in $100-\mathrm{ml}$ round-bottom flask and with $50 \mathrm{ml}$ of methanol as reaction medium. The resultant clear mixture evaporated under vacuum and dried residue was placed in desiccators overnight and stored at room temperature in an amber-colored glass bottle.

\section{Refluxing with Dichloromethane (Salting-Out Method) (20)}

Specific amounts of extracts $(0.33 \mathrm{~g}$ orange peel extract and $0.66 \mathrm{~g}$ liquorice extract) and phospholipid $(1 \mathrm{~g})$ were refluxed at $50^{\circ} \mathrm{C}$ for $3 \mathrm{~h}$ in $100-\mathrm{ml}$ round-bottom flask and with $50 \mathrm{ml}$ of dichloromethane as reaction medium. The resultant clear mixture was evaporated, and $20 \mathrm{ml}$ of $n$-hexane was added to it with stirring. The precipitate was filtered and dried under and dried residue placed in desiccators overnight and stored at room temperature in an amber-colored glass bottle.

\section{Film Formation Method $(19,21)$}

Specific amounts of extract $(0.33 \mathrm{~g}$ orange peel extract and $0.66 \mathrm{~g}$ liquorice extract) and phospholipid (1 g) were taken into a 100-ml round-bottom flask and dispersed in $30 \mathrm{ml}$ methanol. The dispersion was subsequently gently stirred in a warm water bath $\left(45-55^{\circ} \mathrm{C}\right)$, and the formed solution was then continually heated $\left(45-55^{\circ} \mathrm{C}\right)$ with the use of a rotary evaporator to evaporate methanol. The obtained phytophospholipid complex film was further dried overnight in an oven at room temperature and stored in a desiccator until use.

\section{Optimization of Extract:Phospholipid Ratio}

The complexes were prepared with extract (orange peel extract and liquorice extract) and phospholipids in ratio of 1:1, $1: 2,1: 3$, and $2: 1$, respectively. The complexes were then compared based on entrapment efficiency.

\section{Entrapment Efficiency (22)}

Entrapment efficiency (EE) was measured using 1800 UV-visible spectrophotometer (Shimadzu). A known amount of the prepared complex was ultracentrifuged at $5000 \mathrm{rpm}$ for $15 \mathrm{~min}$ in methanol. The amount of phenolic compounds in the supernatant was then analyzed by UV-visible spectrophotometer at $\lambda_{\max } 765 \mathrm{~nm}$; the concentration of phenolic compounds was measured. All measurements were performed in triplicate.

The EE was calculated using the following equation:

$\mathrm{EE}(\%)=(T-S) / T \times 100$

Where,

$T$ Total amount of phenolic compounds present in quantity of phytophospholipid complex taken

$S$ Amount of phenolic compound in the supernatant

$T-S$ Amount of phenolic compounds entrapped

\section{Evaluation of Phytophospholipid Complex}

The optimized complex was further evaluated using following parameters.

Scanning Electron Microscopy. Samples were studied using ZEISS Ultra-55 Scanning Electron Microscope at $5 \mathrm{kV}$. Suspension of phytophospholipid complex with water was coated on silicon wafer. It was dried and surface morphology was viewed and photographed with scanning electron microscope

Fourier Transform Infrared Spectrophotometry. Fourier transform infrared spectrophotometry (FT-IR Spectrometer, BRUKER IFS-55, Switzerland) was employed to study the interaction between extracts and phospholipids and to ascertain the structure and chemical stability of phytophospholipid complex, phospholipid, and extracts. The IR spectra of extracts, phospholipids, their complex, and physical mixture were obtained by the potassium bromide $(\mathrm{KBr})$ method. $\mathrm{KBr}$ pellets were prepared by gently mixing 1-mg sample with $100 \mathrm{mg}$ KBr. Spectral 
scanning was done in the range between 4000 and $400 \mathrm{~cm}^{-1}$.

Differential Scanning Calorimetry. Orange peel extract, liquorice extract, phospholipid, physical mixture of extracts and phospholipid, and phytophospholipid complex were placed in the aluminum crimp cell and heated at the speed of $10^{\circ} \mathrm{C} \mathrm{min}^{-1}$ from 0 to $300^{\circ} \mathrm{C}$ in the atmosphere of nitrogen. The peak transition onset temperatures were recorded by means of an analyzer.

\section{Development of Cream Containing Orange Peel-Liquorice- Phospholipid Complex}

Ingredients listed in Table I were accurately weighed and placed in separate beakers (for oil phase and water phase). The beakers were heated to $70^{\circ} \mathrm{C}$ on a water bath. On complete melting of the ingredients, the contents of the water phase were added to the oil phase with constant stirring. The resulting emulsion was stirred until it cooled and congealed. One percent phytophospholipid complex was incorporated into the base by shear.

Conventional ( $1 \%$ ) extract cream was prepared by incorporating desired quantity of the extract in the water phase and then adding it to the oil phase with stirring using the same method as above. Cream base in which phytophospholipid complex was stable was further evaluated and kept for stability studies.

\section{Evaluation of Cream}

Organoleptic Properties. Appearance and emolliency of cream were checked.

Presence of Foreign Particles/Grittiness. A small amount of cream was taken and spread on a glass slide free from grease and was observed against diffused light to check for presence of foreign particles.

$p H$. The $\mathrm{pH}$ meter was calibrated using standard buffer solution. About $0.5 \mathrm{~g}$ of the cream was weighed and dissolved in $50 \mathrm{ml}$ of distilled water, and its $\mathrm{pH}$ was measured.

\section{Rheological Properties.}

- Viscosity (23) - viscosity of the formulation was determined by Brookfield viscometer using spindle no 18.

- Spreadability (24) - spreadability is a term expressed to denote the extent of area to which the topical formulation spreads on application to skin. Spreadability of cream was measured with the glass slide apparatus.

$$
s=w^{*} l / t
$$

where

$S$ Spreadability $\left(\mathrm{g} \mathrm{cm} \mathrm{s}^{-1}\right)$

$w$ Weight on upper slide $(\mathrm{g})$

$l$ Length of slide $(\mathrm{cm})$

$t$ Time (s)

\section{Drug Content (25)}

One gram of cream was dissolved in $30 \mathrm{ml}$ methanol, and solution was kept aside intact for half an hour to attain homogenous equilibrium. The solution was withdrawn, the Abs was estimated by UV spectrometer at $765 \mathrm{~nm}$, and the concentration of phenolic compounds was measured. The experiment was performed in triplicate.

Anti-Oxidant Activity (26). One gram of cream was weighed and mixed with $10 \mathrm{ml}$ of methanol and sonicated for $30 \mathrm{~min}$. It was centrifuged at $5000 \mathrm{rpm}$ at $4^{\circ} \mathrm{C}$ for $20 \mathrm{~min}$, and solution was filtered through $0.45-\mu \mathrm{m}$ nylon syringe filter. Antioxidant activity of filtrate was determined using DPPH radical scavenging assay. The experiment was performed in triplicate.

Ex Vivo Diffusion Studies. Diffusion of topical formulation was performed using biological membrane of pig ear and modified Franz's diffusion cells. The skin was stored in a refrigerator at $-20^{\circ} \mathrm{C}$ in phosphate buffer. The receptor

Table I. Composition of Creams

\begin{tabular}{|c|c|c|c|c|c|c|c|c|c|c|}
\hline Ingredient & 1 & 2 & 3 & 4 & 5 & 6 & 7 & 8 & 9 & Mode of action \\
\hline Stearic acid & 24 & 13 & 4 & 10 & 20 & 20 & 20 & 20 & 20 & Stiffening agent \\
\hline Mineral oil & - & - & - & 3.5 & - & - & - & - & - & Solvent \\
\hline Isopropyl myristate & - & - & - & - & 20 & 2 & - & - & - & Emollient \\
\hline Triethanolamine & - & - & - & - & 1.2 & 1.2 & 1.2 & 1.2 & 1.2 & Neutralizer \\
\hline Petroleum jelly & - & - & 1 & - & - & - & 2 & 2 & 2 & Emollient \\
\hline Cetyl alcohol & - & 1 & 5 & 2 & - & - & - & - & - & Stiffening agent \\
\hline Potassium hydroxide & 1 & 0.9 & 1 & - & - & - & - & - & - & Soap formation \\
\hline Glycerin & 11 & 10 & 5 & - & - & - & - & - & - & Solvent \\
\hline Glyceryl monostearate & - & 1 & - & - & - & - & - & - & - & Emulsifier \\
\hline Sodium metabisulfite & - & - & - & 0.1 & - & - & - & - & - & Preservative \\
\hline Ethylenediaminetetraacetic acid (EDTA) & - & - & - & 0.1 & - & - & - & - & - & Chelating agent \\
\hline Methylparaben & 0.18 & 0.18 & 0.18 & 0.18 & 0.18 & 0.18 & 0.18 & 0.18 & 0.18 & Preservative \\
\hline Propylparaben & 0.02 & 0.02 & 0.02 & 0.02 & 0.02 & 0.02 & 0.02 & 0.02 & 0.02 & Preservative \\
\hline Water & q.s. & q.s. & q.s. & q.s. & q.s. & q.s. & q.s. & q.s. & q.s. & Solvent \\
\hline Phytophospholipid complex & 1 & 1 & 1 & 1 & 1 & 2 & 1 & 2.5 & 5 & Anti-oxidant, anti-aging \\
\hline
\end{tabular}

q.s. quantity sufficient 
compartment was filled with $11 \mathrm{ml}$ of phosphate buffer solution, and the donor chamber contained $1 \mathrm{~g}$ of each formulation. It was covered with parafilm to prevent evaporation. The receptor solution was continuously stirred at $210 \mathrm{rpm}$. The temperature was maintained at $37^{\circ} \mathrm{C}$ by circulating water through an external water jacket and protected from light with aluminum foil. All determinations were performed in triplicate. One milliliter of aliquot solution in the receptor chambers was withdrawn through the sampling port at $0.5,1,2,3,6$, 9,12 , and $24 \mathrm{~h}$, and cells were refilled with fresh phosphate buffer solution so as to keep the volume of receptor solution constant during the experiment. The solution in receptor compartment was analyzed spectrophotometrically using 1800 UV-visible spectrophotometer (Shimadzu) at $\lambda_{\max } 765 \mathrm{~nm}$; the concentration of phenolic compounds diffused through pig skin membrane was measured. Comparison between diffusion profiles of phytophospholipid cream and extract cream was done.

Analysis of Phenolic Compounds Retained in Skin. At the end of the ex vivo diffusion experiment, phenolic compounds retained in the skin were extracted by cutting the pig skin into small pieces with the scissors and soaking them in $5 \mathrm{ml}$ methanol. It was homogenized at 24,000 rpm for $5 \mathrm{~min}$. The small pieces of pig skin were filtered using filter paper. The filtrate was centrifuged at $15,000 \mathrm{rpm}$ for $30 \mathrm{~min}$ at $4^{\circ} \mathrm{C}$. The amount of phenolic compounds retained in the skin was measured using $1800 \mathrm{UV}$-visible spectrophotometer (Shimadzu) at $\lambda_{\max } 765 \mathrm{~nm}$. All determinations were performed in triplicate.

Stability Studies. To assess formulation stability, stability studies were done according to ICH guidelines. The cream was filled in bottle and kept in humidity chamber maintained at $30 \pm 2^{\circ} \mathrm{C} / 65 \pm 5 \% \mathrm{RH}$ (relative humidity) and $40 \pm 2{ }^{\circ} \mathrm{C} / 75 \pm 5 \%$ $\mathrm{RH}$ and also at $25^{\circ} \mathrm{C}$ (RT-room temperature) for 3 months. Creams were analyzed for appearance, phase separation, $\mathrm{pH}$, viscosity, spreadability, drug content, and anti-oxidant activity at $0,1,2$, and 3 months. The experiment was performed in triplicate.

Skin Irritation Test (27). It was carried out by Draize patch test on six Wistar rats. Healthy Wistar albino rats of either sex and of approximately the same age, weighing about 180-230 g, were used for the study. Animals were acclimatized for 7 days before experimentation. They were fed a standard diet and water ad libitum and were housed in polypropylene cages maintained under standard conditions of 12-h light and dark cycles at $23 \pm 2^{\circ} \mathrm{C}$ temperature. Animal experiments were conducted as per the institute's guidelines of the animal ethics committee. The experimental protocol was approved by Institutional Animal Ethical Committee of Dr. Bhanuben Nanavati College of Pharmacy (CPCSEA/ IAEC/BNCP/P-11/2014). Hair was removed from the backside of rats, and an area of $1 \mathrm{~cm}^{2}$ was marked on both the sides. One side served as control while the other as test. Creams were applied (200 mg/rat), and the site was covered with a cotton bandage. The applied formulation was removed, and the skin was observed for any visible change such as erythema (redness), at 24, 48, and $72 \mathrm{~h}$ after application of formulation.
In Vitro Screening of the Polyherbal Phytophospholipid Cream for Anti-Elastase Activity (28)

The study was performed in full compliance with the SRC (Scientific Research Centre) guidelines for Anti-Elastase Activity as per Protocol Number SRC/BT/5401-10.

Test procedure for anti-elastase activity determination

- The test sample was prepared in buffer [0.2 M Tris- $\mathrm{HCl}$ buffer ( $\mathrm{pH}$ 8.0)]. The enzyme was added to the test and incubated for $15 \mathrm{~min}$. The reaction was initiated by adding substrate.

- Each test reaction was incubated for $15 \mathrm{~min}$ at $25^{\circ} \mathrm{C}$, and the Abs was read at $410 \mathrm{~nm}$. The control consisted on only the buffer without any test sample.

- Appropriate blanks were run. Two independent experiments (in triplicate) were conducted to study the antielastase activity of the test sample. The results were expressed as mean \pm standard deviation.

- The following formula was used for calculating \% Elastase inhibition

$$
\% \text { Elastase inhibition }=100-\frac{\text { Absorbance of test }}{\text { Absorbance of control }} \times 100
$$

\section{RESULTS AND DISCUSSION}

\section{Preparation of Crude Extract}

The methanolic extract of orange peel and liquorice gave a dark-yellow and dark-brown extract, respectively.

\section{Total Phenolic Content}

The TPC in methanolic extracts of orange peel and liquorice determined by the Folin-Ciocalteu method is expressed in terms of gallic acid equivalent (the standard curve equation: $y=0.0075 x-0.0062, r^{2}=0.9979$ ). The values obtained for the concentration of total phenols are expressed as milligram of GAE per gram of extract.

\section{Total Flavonoid Content}

The concentration of flavonoids in methanolic extracts of orange peel and liquorice was determined using spectrophotometric method with aluminum chloride. The content of flavonoids was expressed in terms of rutin equivalent (the standard curve equation: $\left.y=0.0015 x+0.0027, r^{2}=0.9997\right)(\mathrm{mg}$ of RUE $\mathrm{g}^{-1}$ of extract).

\section{Assessment of Anti-Oxidant Activity}

The extracts were capable of scavenging hydrogen peroxide and inhibiting DPPH in a concentration-dependent manner. The anti-oxidant activity of the extract was expressed as $\left(\mathrm{IC}_{50}\right)$, which was defined as the concentration $\left(\mu \mathrm{g} \mathrm{ml}^{-1}\right)$ of extract required to scavenge $50 \%$ of radicals. The $\mathrm{IC}_{50}$ of orange peel and liquorice extract in $\mathrm{H}_{2} \mathrm{O}_{2}$ of scavenging assay was $60.51 \pm 0.44$ and $46.66 \pm 0.65 \mu \mathrm{g} \mathrm{ml}^{-1}$, respectively, in comparison to standard ascorbic acid $36.41 \pm 0.09 \mu \mathrm{g} \mathrm{ml}^{-1}$, and $\mathrm{IC}_{50}$ 
Table II. Consolidated Data of Chemical Analysis and Anti-oxidant Assays of Methanolic Extracts of Orange Peel and Liquorice

\begin{tabular}{|c|c|c|c|c|}
\hline \multirow[t]{2}{*}{ Sample } & \multicolumn{2}{|c|}{$\mathrm{IC}_{50}\left(\mu \mathrm{g} \mathrm{ml}^{-1}\right)$} & \multirow{2}{*}{$\begin{array}{l}\text { Total phenolic content }(\mathrm{mg}) \\
\qquad \text { GAE g }^{-1} \text { extract }\end{array}$} & \multirow{2}{*}{$\begin{array}{l}\text { Total flavonoid content }(\mathrm{mg}) \\
\text { RUE } \mathrm{g}^{-1} \text { extract }\end{array}$} \\
\hline & DPPH assay & $\mathrm{H}_{2} \mathrm{O}_{2}$ assay & & \\
\hline Orange peel extract & $60.37 \pm 0.15$ & $60.51 \pm 0.44$ & $391.2 \pm 1.64$ & $116.23 \pm 0.68$ \\
\hline Liquorice extract & $48.23 \pm 0.28$ & $46.66 \pm 0.65$ & $518.9 \pm 2.54$ & $233.67 \pm 2.56$ \\
\hline Ascorbic acid (reference standard) & $34.99 \pm 0.24$ & $36.41 \pm 0.09$ & - & - \\
\hline
\end{tabular}

Each value in the table was obtained by calculating the mean $\pm \mathrm{SD}(n=3)$

$I C_{50}$ The concentration of extract required to scavenge $50 \%$ of the DPPH radical $\mathrm{DPPH}$ 2,2-diphenyl-1-picrylhydrazyl, $\mathrm{H}_{2} \mathrm{O}_{2}$ hydrogen peroxide, $G A E$ gallic acid equivalent, $R U E$ rutin equivalent

of orange peel and liquorice extract in DPPH assay was 60.37 \pm 0.15 and $48.23 \pm 0.28 \mu \mathrm{g} \mathrm{ml}^{-1}$ when compared to standard ascorbic acid $34.99 \pm 0.24 \mu \mathrm{g} \mathrm{ml}^{-1}$. The observed anti-oxidant effect can be attributed majorly to the phenolic compounds.

Consolidated results of TPC, TFC, and anti-oxidant assays of methanolic extracts of orange peel and liquorice are tabulated in Table II.

\section{Correlation Studies}

Results of correlation studies are tabulated in Table III. A significant correlation $(r=0.918, p<0.01)$ between TPC and DPPH scavenging activity was observed. Also, a significant correlation $(r=0.835, p<0.01)$ was seen between TPC and $\mathrm{H}_{2} \mathrm{O}_{2}$ scavenging activity. This indicates that the anti-oxidant activity of the extracts of orange peel and liquorice is due to their phenolic constituents. These results are in accordance with other reports in the literature, which showed positive strong correlation between anti-oxidant activities and TPC (29). Recent investigations have shown that many flavonoids and related polyphenols contribute significantly to the antioxidant activity of medicinal plants (30). TFC showed good correlation with both DPPH scavenging activity $(r=0.845$, $p<0.05)$ and $\mathrm{H}_{2} \mathrm{O}_{2}$ scavenging activity $(r=0.725, p<0.05)$.

\section{Combination Studies}

Results of TPC, TFC, and anti-oxidant activity (DPPH scavenging activity and $\mathrm{H}_{2} \mathrm{O}_{2}$ scavenging activity) carried out on different ratios of orange peel extract and liquorice extract are consolidated in Table IV. As evident from the results, ratio 1:2 (orange peel (OP)/Liq) gave maximum TPC, TFC, DPPH

Table III. Pearson Correlation Coefficient Between the Anti-oxidant Activities and Total Phenolic Content and Total Flavonoid Content

\begin{tabular}{lcc}
\hline Anti-oxidant assay & \multicolumn{2}{c}{ Pearson correlation coefficient $(r)$} \\
\cline { 2 - 3 } & $\begin{array}{l}\text { Total phenolic } \\
\text { content (TPC) }\end{array}$ & $\begin{array}{l}\text { Total flavonoid } \\
\text { content (TFC) }\end{array}$ \\
\hline $\begin{array}{l}\text { DPPH scavenging } \\
\text { activity }\end{array}$ & 0.918 & 0.845 \\
$\mathrm{H}_{2} \mathrm{O}_{2}$ scavenging & 0.835 & 0.725 \\
activity & & \\
\hline
\end{tabular}

DPPH 2,2-diphenyl-1-picrylhydrazyl, $\mathrm{H}_{2} \mathrm{O}_{2}$ hydrogen peroxide scavenging activity, and $\mathrm{H}_{2} \mathrm{O}_{2}$ scavenging activity. Therefore, 1:2 ratio was used for further work.

\section{Complex Preparation}

The work by Bombardelli and Patri referred to solvent evaporation as a possible method for complex formation (31). Solvent evaporation technique was applied in this study using methanol as a volatile solvent. For preparation of phytophospholipid complexes, obtaining a clear drug-phospholipid solution was mandatory (19). Clear solution of orange peel-liquorice extract and phospholipid could be obtained in this solvent. The yield of phytophospholipid complex formed by this method was $69 \%$. On the other hand, the yield of phytophospholipid complex formed by film formation method was $44 \%$. Also, upon addition of $n$-hexane in salting-out method, frothing was observed. The precipitate was observed in minute quantity. Thus, solvent evaporation method using methanol was found to be suitable for formation of phytophospholipid complex of orange peel-liquorice.

\section{Optimization of Extract:Phospholipid Ratio}

Four extract:phospholipid ratios (1:1, 1:2, 1:3, 2:1) were prepared and evaluated. The phytophospholipid complexes were then compared based on EE as depicted in Table V. Results showed that complex with ratio 1:1 showed highest EE. Therefore, 1:1 was selected as optimum ratio for further characterization of complex and formulation of cream.

\section{Evaluation of Phytophospholipid Complex}

The optimized complex was further evaluated using following parameters.

\section{Scanning Electron Microscopy}

The surface morphology phytophospholipid complex as examined by scanning electron microscopy (SEM) at various magnifications is shown in Fig. 1. Phytophospholipid complex was made up of phospholipids and extract and appeared irregular sphere shape. The vesicles consisted of phospholipids, and extract was intercalated in lipid layer.

\section{Fourier Transform Infrared Spectrophotometry}

FT-IR was used to confirm the interaction between extracts and phospholipids in phytophospholipid complex (Fig. 2). In FT-IR spectra of extracts, the band at 3432 and 
Table IV. Consolidated Data of TPC, TFC, and Anti-Oxidant Assays of Ratios of Methanolic Extracts of Orange Peel and Liquorice

\begin{tabular}{ccccc}
\hline Ratio (OP extract:LE) & TPC (mg GAE g ${ }^{-1}$ extract) & TFC (mg RUE g ${ }^{-1}$ extract) & DPPH (\% inhibition) & $\mathrm{H}_{2} \mathrm{O}_{2}(\%$ inhibition $)$ \\
\hline $1: 1$ & $460.71 \pm 0.32$ & $153.78 \pm 2.63$ & $68.37 \pm 0.07$ & $61.59 \pm 0.37$ \\
$1: 2$ & $530.00 \pm 1.56$ & $246.25 \pm 1.03$ & $87.99 \pm 0.64$ & $72.47 \pm 0.86$ \\
$2: 3$ & $480.40 \pm 2.38$ & $173.52 \pm 0.46$ & $70.74 \pm 2.13$ & $67.24 \pm 1.61$ \\
$3: 2$ & $474.30 \pm 0.96$ & $164.25 \pm 3.46$ & $66.54 \pm 1.29$ & $55.29 \pm 2.47$ \\
$2: 1$ & $431.50 \pm 2.15$ & $132.2 \pm 0.97$ & $59.16 \pm 0.79$ & $48.93 \pm 1.56$ \\
$1: 0$ & $391.20 \pm 1.64$ & $116.23 \pm 0.68$ & $43.37 \pm 0.65$ & $37.46 \pm 2.16$ \\
$0: 1$ & $518.90 \pm 2.54$ & $233.67 \pm 2.56$ & $69.27 \pm 3.61$ & $56.51 \pm 0.67$ \\
\hline
\end{tabular}

Each value in the table was obtained by calculating the mean $\pm \mathrm{SD}(n=3)$

$T P C$ total phenolic content, $T F C$ total flavonoid content, $O P$ orange peel, $L E$ liquorice extract, $G A E$ gallic acid equivalent, $R U E$ rutin equivalent, $D P P H$ 2,2-diphenyl-1-picrylhydrazyl, $\mathrm{H}_{2} \mathrm{O}_{2}$ hydrogen peroxide

$3378 \mathrm{~cm}^{-1}$ was attributed to the stretching vibration of the phenolic $\mathrm{OH}$ group in orange peel extract and liquorice extract, respectively. Additionally, sharp absorption bands were observed at 1611 and $1600 \mathrm{~cm}^{-1}$ (stretching vibrations of a benzene ring) in orange peel extract and liquorice extract, respectively. The strong peaks at 1728 and $1224 \mathrm{~cm}^{-1}$ in phospholipids were due to $\mathrm{C}=\mathrm{O}$ absorption and $\mathrm{P}=\mathrm{O}$ absorption, respectively. The strong peaks at 2918 and $2848 \mathrm{~cm}^{-1}$ in phospholipids could be due to stretching and deformation of methyl groups. The peak at $1454 \mathrm{~cm}^{-1}$ observed in phospholipids could be due to bending vibration of $\mathrm{CH}_{2}$. $\mathrm{P}-\mathrm{O}-\mathrm{C}$ stretching band at $1073 \mathrm{~cm}^{-1}$ was also observed in phospholipids. It was obvious that the physical mixture and the complex indicated distinct IR spectra. The spectrum of the physical mixture showed an additive effect of extracts and phospholipids, in which the characteristic absorption peaks of extracts were still present at $3378 \mathrm{~cm}^{-1}, 2313 \mathrm{~cm}^{-1}$ (liquorice), and $1611 \mathrm{~cm}^{-1}$ (orange peel). However, shift of absorption peak of phenolic OH stretching from $3432 \mathrm{~cm}^{-1}$ (OP) and $3378 \mathrm{~cm}^{-1}$ (Liq) to $3350 \mathrm{~cm}^{-1}$ (lower frequency) compared with extracts and a shift from 1224 to $1225 \mathrm{~cm}^{-1}$ ( $\mathrm{P}=\mathrm{O}$ absorption) and 1073 to $1046 \mathrm{~cm}^{-1}$ (P-O-C stretching band) compared with phospholipids were exhibited by phytophospholipid complex. Additionally, the peak at $3350 \mathrm{~cm}^{-1}$ became remarkably broader. These changes indicated that extract and phospholipids formed a complex by hydrogen bonding between the $\mathrm{OH}$ group of the phenol rings of extracts and the $\mathrm{P}=\mathrm{O}$ group of the phospholipids. The interaction of extracts with the polar part of the phospholipids makes the long hydrocarbon tail of the latter bend freely and envelope its own polar head. Thus, the sequence of the phospholipids is interrupted (19).

\section{Differential Scanning Calorimetry}

Figure 3 shows the differential scanning calorimetry (DSC) thermograms of orange peel extract, liquorice extract,

Table V. Evaluation of Phytophospholipid Complexes

\begin{tabular}{cc}
\hline Ratio (extract:phospholipid) & Entrapment efficiency (\%) \\
\hline $1: 1$ & $93.22 \pm 0.26$ \\
$1: 2$ & $76.23 \pm 0.38$ \\
$1: 3$ & $81.56 \pm 1.08$ \\
$2: 1$ & $72.45 \pm 2.92$
\end{tabular}

Each value in the table was obtained by calculating the mean \pm SD $(n=3)$ phospholipids, physical mixture, and phytophospholipid complex. Phospholipids showed two endothermal peaks, and the first endothermal peak appeared at $170.0^{\circ} \mathrm{C}$. It was considered that the formation of this peak was due to hot movements of phospholipid molecule polarity parts. The second endothermal peak at $175.6^{\circ} \mathrm{C}$ appears sharp-pointed; it was considered that owing to the transition from gel state to liquid crystal state, the carbon-hydrogen chain in phospholipids

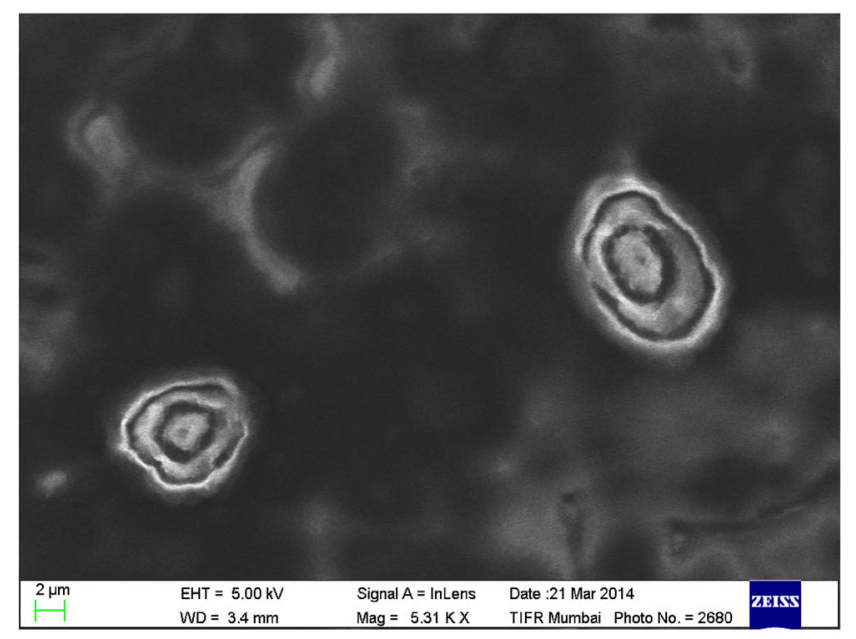

a

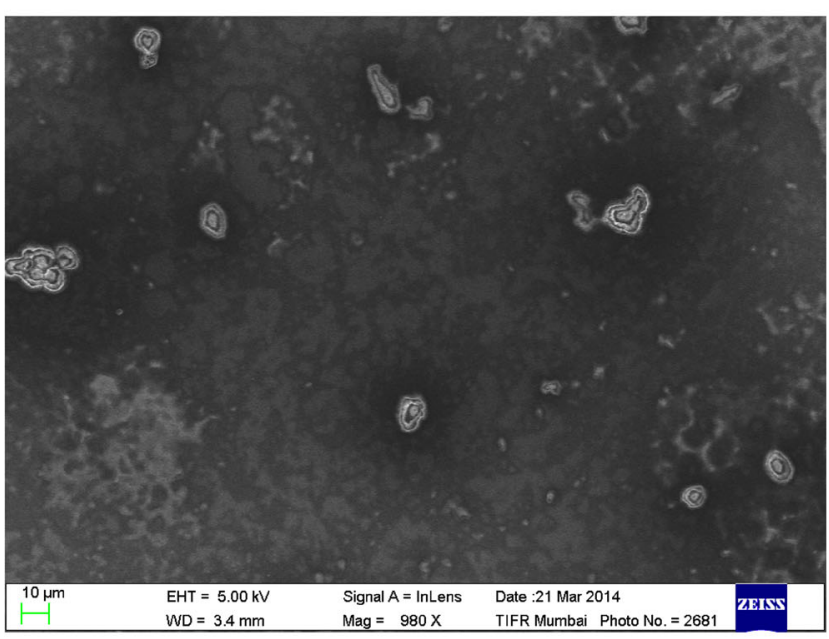

b

Fig. 1. Scanning electron micrographs of phytophospholipid complex 


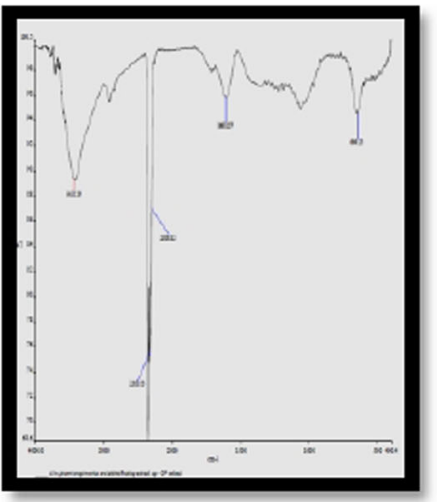

a

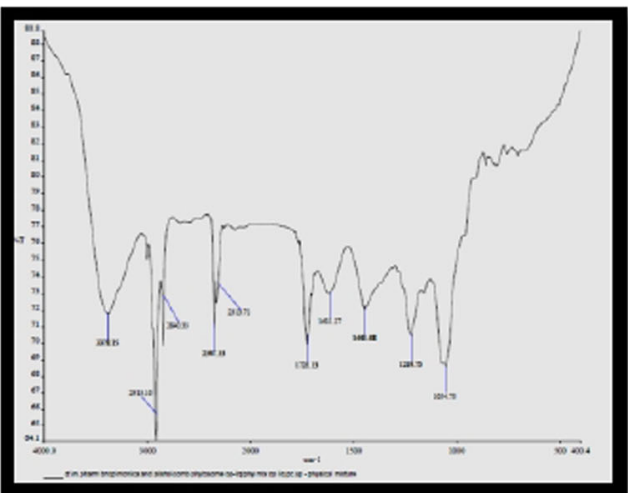

d

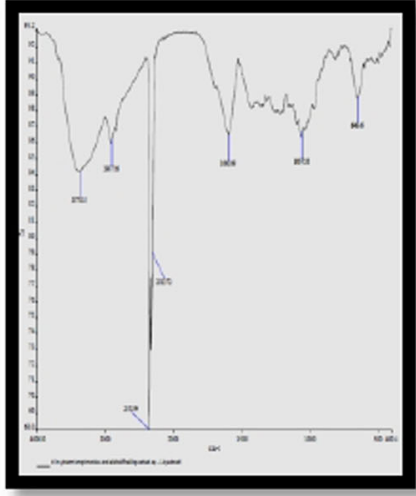

b

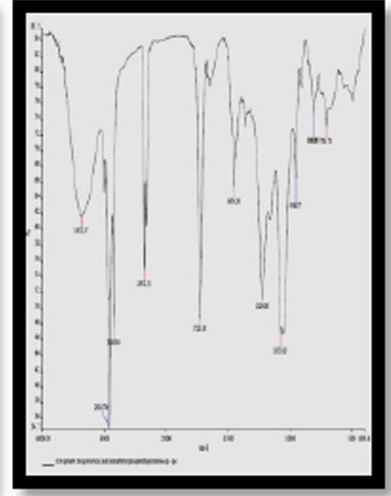

C

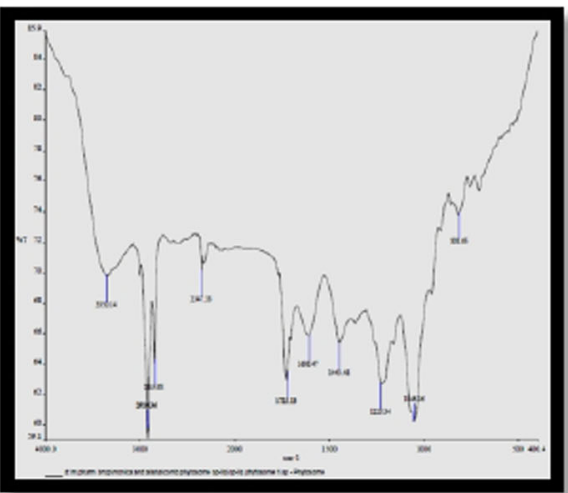

e

Fig. 2. FT-IR spectra of a orange peel extract, $\mathbf{b}$ liquorice extract, $\mathbf{c}$ phospholipid, $\mathbf{d}$ physical mixture, and $\mathbf{e}$ phytophospholipid complex

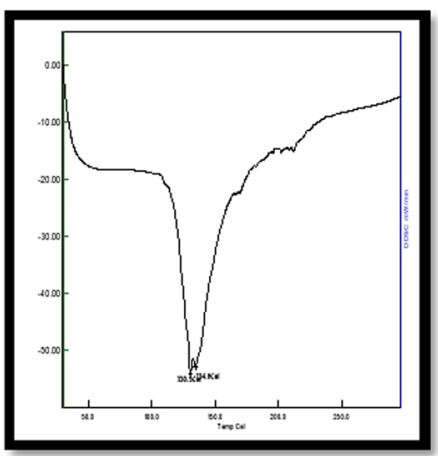

a

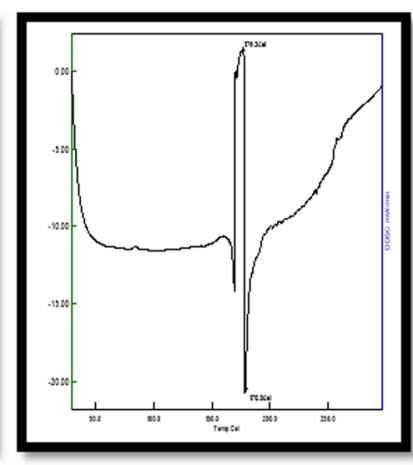

b

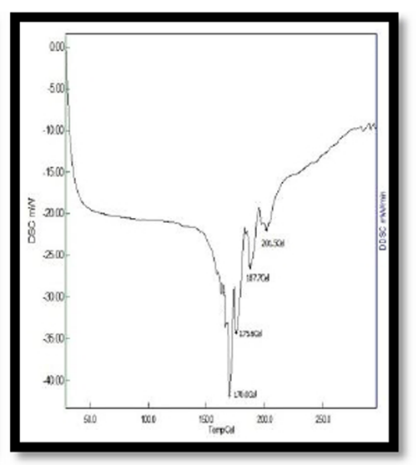

C

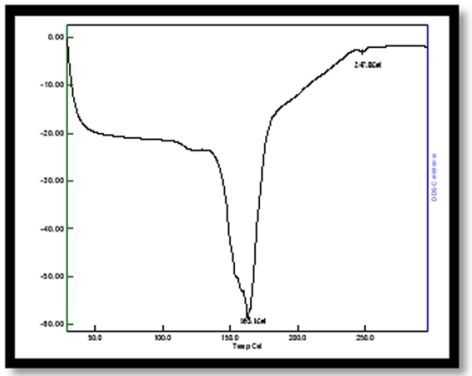

e

Fig. 3. DSC thermograms of a orange peel extract, b liquorice extract, $\mathbf{c}$ phospholipid, d physical mixture, and e phytophospholipid complex 
perhaps happened to melt, isomerous, or the crystal changes. As extracts were not pure, orange peel showed endothermal peaks at 130.5 and $134.6^{\circ} \mathrm{C}$. Liquorice extract showed one exothermal peak at $176.3^{\circ} \mathrm{C}$ and one endothermal peak at $178.8^{\circ} \mathrm{C}$. Physical mixture of extracts and phospholipids showed that there were three endothermal peaks. The first one at $132.9^{\circ} \mathrm{C}$ was a broad peak, coordinating with the endothermal peak of orange peel extract; the endothermal peak at $176.2^{\circ} \mathrm{C}$ could be due to endothermal peak of liquorice extract and second endothermal peak of phospholipids; the third one was at 190.6 in parallel with the endothermal peak of phospholipid. The DSC curve of the physical mixture showed an additive effect of extracts and phospholipid. On the contrary, the DSC curve of the phytophospholipid complex showed that the original peaks of extract and phospholipids disappeared from the thermogram of complex whereas a single endothermal peak at $163.1^{\circ} \mathrm{C}$ different from the peaks of the individual components of the complex was observed. Thus, it can be concluded that extracts might have interacted with phospholipid and van der Waals forces, or hydrogen bonding, singly or in combination might have formed (19).

\section{Development of Cream Containing Orange Peel-Liquorice- Phospholipid Complex}

Various formulations tried for development of stable cream are shown in Table I. Phytophospholipid complex was added in oil phase and heated. Upon addition of water phase, the color changed to green. This is possibly due to instability of phytophospholipid complex at temperatures exceeding 40$45^{\circ} \mathrm{C}$. Therefore, phytophospholipid complex was added after temperature of cream drops to $40^{\circ} \mathrm{C}$ with constant stirring. Phytophospholipid complex (5\%, batch 9 ) could not be incorporated completely in cream base. Batches 7 and 8 showed good physical appearance, smoothness, stability on storage, and ease of application. Anti-oxidant activity (DPPH scavenging activity) of batch $7(95.96 \% \pm 0.897)$ was more than that for batch $8(92.46 \% \pm 0.065)$. Hence, batch 7 was selected for further evaluation and stability studies.

Thus, phytophospholipid complex system can be beneficial in topical cosmetic formulation, as it is responsible for retention of cream in skin leading to prolonged and stronger topical action. In case of phytophospholipid complex, retention of phytoconstituents in skin for longer time (due to higher affinity of the complex for skin phospholipids) compared to the free active principles is observed. This increases the duration of the anti-oxidant activity on the skin cells as the complex slowly releases the active principle $(32,33)$. Phospholipid helps to nourish skin by improving its hydration and maintains integrity of the collagen structure, further contributing to its healthy appearance.

\section{Evaluation of Optimized Cream}

The optimized formulation (batch 7) was evaluated for various parameters like appearance, grittiness, $\mathrm{pH}$, viscosity, spreadability, drug content, anti-oxidant activity, ex vivo diffusion studies and stability studies for 3 months, and skin irritation studies.
The cream was pale yellow in color with a characteristic pleasant odor. There were no foreign particles in the cream. Also, the cream was smooth in texture and there was no grittiness. This was confirmed by visual appearance and by touch. The $\mathrm{pH}$ of the cream was found to be $7.2 \pm 0.012$ which is good for topical application as skin pH ranges from 4 to 7 . Viscosity of cream is found to be different at different revolution per minute. At 2.5 to $100 \mathrm{rpm}$, viscosity was decreased from 1098 to $27.7 \mathrm{cp}$. So, if we decrease the rate of shear, it increases the viscosity of cream. Viscosity of creams is inversely proportional to rate of shear (rpm). The formulated cream showed good spreadability. It was found to be 12.5 $\pm 0.452 \mathrm{~g} \mathrm{~cm} \mathrm{~s}^{-1}$. The drug content of cream (phenolic content) was found to be $97.78 \% \pm 0.473$. The anti-oxidant activity (DPPH scavenging activity) of cream was found to be $95.96 \% \pm 0.897$.

Skin irritation study conducted on Wistar albino rats when observed visually for sensitivity and reaction at intervals of 24,48 , and $72 \mathrm{~h}$ showed absence of erythema.

In stability studies, all the parameters like appearance, phase separation, $\mathrm{pH}$, viscosity, spreadability, drug content, and anti-oxidant activity did not show significant variation over the period of 3 months. There was no phase separation in 3 months.

\section{Ex Vivo Diffusion Studies}

The percentage of phenolic compounds diffused through the membrane can be directly related to the percentage of cream diffused through membrane. From the release profiles of extract cream and phytophospholipid cream (Fig. 4), it can be concluded that small fraction of phytophospholipid complex cream diffused through the pig skin membrane at the end of $24 \mathrm{~h}$ in contrast to extract cream which was diffused very fast. Besides determination of phenolic compounds in the receptor compartment, the amount of the phenolic compounds retained in skin at the end of $24 \mathrm{~h}$ was also calculated. Higher amounts of phenolic compounds were retained in pig skin in case of phytophospholipid cream $(33.89 \pm 2.46 \mu$

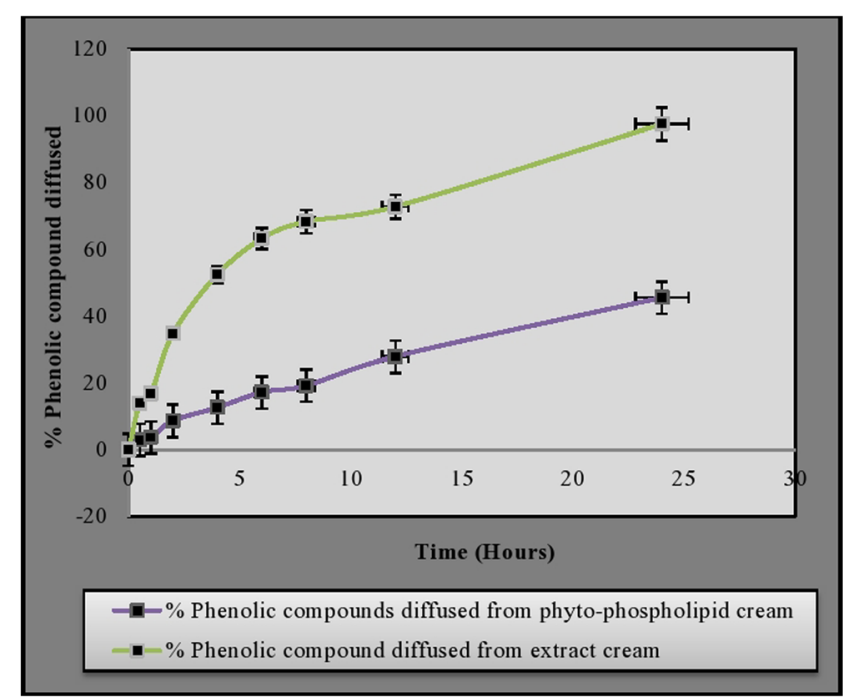

Fig. 4. Percentage of total phenolic compounds diffused from extract cream and phytophospholipid cream 


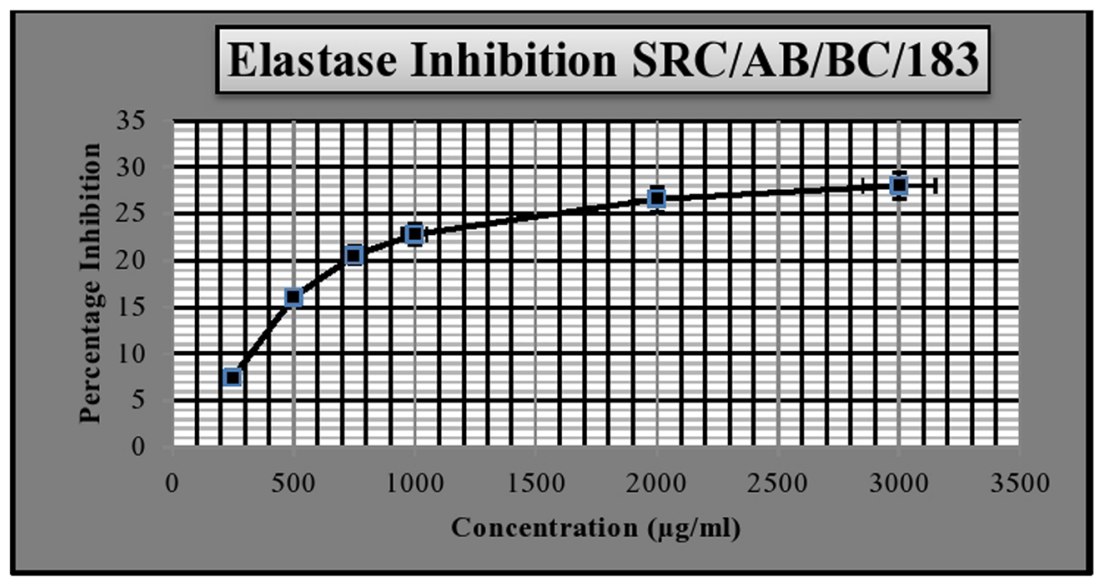

Fig. 5. Elastase inhibition activity of a polyherbal phytophospholipid cream

GAE $\left.\mathrm{cm}^{-2}\right)$ compared to that of extract cream $(5.87 \pm 1.56 \mu$ $\mathrm{GAE} \mathrm{cm}^{-2}$ ).

\section{In Vitro Screening of the Polyherbal Phytophospholipid Cream for Anti-Elastase Activity}

The study was designed to determine the anti-elastase activity (in vitro), in terms of inhibition of the enzyme elastase, by the investigational samples. The polyherbal phytophospholipid cream retained anti-elastase activity of extracts (34). The polyherbal cream showed an increase in elastase inhibition with increase in concentration (Fig. 5). The result (SRC/AB/BC/183) had a mean anti-elastase activity of $28.02 \pm 0.95 \%$ at a concentration of $3000 \mu \mathrm{g} \mathrm{ml} \mathrm{m}^{-1}(w / v)$. The $\mathrm{IC}_{50}$ was not obtained up to $3000 \mu \mathrm{g} \mathrm{ml} \mathrm{m}^{-1}(w / v)$.

\section{CONCLUSION}

Orange peel extract and liquorice extract showed good TPC, TFC, and anti-oxidant activity. By combining these extracts, synergistic anti-oxidant activity was achieved. Solvent evaporation method was chosen for phytophospholipid complex preparation because it gave good yield and EE. Out of the four complexes of extract:phospholipid, ratio 1:1 exhibited highest EE. Evaluation parameters of phytophospholipid complex confirmed formation of complex. Comparison between diffusion profiles and skin retention studies of extract cream and phytophospholipid complex cream showed that there was retention of polyphenols in skin for longer time in phytophospholipid complex cream when compared to extract cream. This could increase the duration of the activity as the complex slowly releases the active principle. Thus, it can be concluded that the phytophospholipid complexes can be a better system for topical delivery of polyphenols in liquorice and orange peel extracts. In vitro screening of the polyherbal phytophospholipid cream for anti-elastase activity showed that the cream retained antielastase activity found in extracts and exhibited a mean anti-elastase activity of $28.02 \pm 0.95 \%$ at a concentration of $3000 \mu \mathrm{g} \mathrm{ml}^{-1}(w / v)$. Thus, phospholipid complex of orange peel and liquorice extracts exhibited good potential as anti-aging cosmeceutical and can either be used alone or as an additive to an anti-aging formulation.

\section{ACKNOWLEDGMENTS}

The authors wish to express their gratitude to VAV Life Sciences Pvt. Ltd., Mumbai, India, for the generous gift of "Leciva-S70" sample. We thank Tata Institute of Fundamental Research, Mumbai, India, for the help rendered in the conduct of scanning electron microscopy analysis. Special thanks go to Kelkar Research Centre, Mumbai, India, for carrying out "in vitro anti-elastase activity" test of the formulation. We are also grateful to SVKM's Dr. Bhanuben Nanavati College of Pharmacy, Mumbai, India, for provision of expertise, technical support, and necessary guidance regarding the project.

\section{REFERENCES}

1. Anti-aging, http://jp.indena.com/pdf/jp/anti_aging.pdf (Date accessed 12 July 2014).

2. Serri R, Iorizzo M. Combating aging skin. Clin Dermatol. 2008;26(2):105.

3. Korać RR, Khambholja KM. Potential of herbs in skin protection from ultraviolet radiation. Pharmacogn Rev. 2011;5(10):164-73.

4. Caragay AB. Cancer-preventive foods and ingredients. Food Technol. 1992;46(4):65-8.

5. Mukherjee PK, Rai S, Kumar V, et al. Plants of Indian origin in drug discovery. Expert Opin Drug Discov. 2007;2:633-57.

6. Yusof S, Mohd Ghazali H, Swee KG. Naringin content in local citrus fruits. Food Chem. 1990;37:113-21.

7. Obolentseva GV, Litvinenko VI, Ammosov AS, et al. Pharmacological and therapeutic properties of licorice preparations (a review). Pharm Chem J. 1999;33:24-31.

8. Visavadiya NP, Soni B, Dalwadi N. Evaluation of antioxidant and anti-atherogenic properties of Glycyrrhiza glabra root using in vitro models. Int J Food Sci Nutr. 2009;60(2):135-49.

9. Ahshawat MS, Saraf S, Saraf S. Preparation and characterization of herbal creams for improvement of skin viscoelastic properties. Int J Cosmet Sci. 2008;30(3):183-93.

10. Rafeeuddin M, Rao NV, Shanta Kumar SM, et al. Comparative efficacy of four Ayurvedic antidiabetic formulations in alloxan-induced diabetic rabbits. Acta Pharma Sci. 2009;51:33-8.

11. Yokomizo Y, Sagitani H. The effects of phospholipids on the percutaneous penetration of indomethacin through the dorsal skin of guinea pig in vitro 2 . The effects of the hydrophobic group 
in phospholipids and a comparison with general enhancers. J Control Release. 1996;42:37-46.

12. Raghavan S. Handbook of spices. Seasonings and flavourings. 2nd ed. Boca Raton: CRC Press, Taylor and Francis Group; 2007.

13. Sasaki H, Kojima M, Nakamura J, et al. Acute toxicity and skin irritation of pyrrolidone derivatives as transdermal penetration enhancer. Chem Pharm Bull. 1990;38:2308-10.

14. Ghosh TK, Pfister WR, Yum SI. Transdermal and topical drug delivery system. Buffalo Grove: Interpharm Press; 1997. p. 191214.

15. Singleton VL, Rossi Jr JA. Colorimetry of total phenolics with phosphomolybdic-phosphotungstic acid reagents. Am J Enol Vitic. 1965;16:144-58.

16. Marinova D, Ribarova F, Atanassova M. Total phenolics and total flavonoids in Bulgarian fruits and vegetables. J Univ Chem Technol Metall. 2005;40(3):255-60.

17. Shirwaikar A, Prabhu KS, Punitha ISR. In-vitro antioxidant studies of Sphaeranthus indicus (Linn). Indian J Exp Biol. 2006;44:993-6.

18. Ruch RJ, Cheng SJ, Klaunig JE. Prevention of cytotoxicity and inhibition of intracellular communication by antioxidant catechins isolated from Chinese green tea. Carcinogenesis. 1989;10:1003-8.

19. Freag MS, Elnaggar Y, Abdallah OY. Lyophilized phytosomal nanocarriers as platforms for enhanced diosmin delivery: optimization and ex vivo permeation. Int J Nanomedicine. 2013;8:238597.

20. Kuntal M, Kakali M, Arunava G, et al. Curcumin-phospholipid complex: preparation, therapeutic evaluation and pharmacokinetic study in rats. Int J Pharm. 2007;330:155-63.

21. Jain NK. Liposomes as drug carriers. Controlled and novel drug delivery. 1st ed. New Delhi: CBS Publisher; 2005. p. 308.

22. Xia HJ, Zhang ZH, Jin X, et al. A novel drug phospholipid complex enriched with micelles: preparation and evaluation in vitro and in vivo. Int J Nanomedicine. 2013;8:545-54.
23. Demiana NI. Formulation and evaluation of itraconazole via liquid crystal for topical delivery system. J Pharm Biomed Anal. 2001;26:387-99.

24. Shrikhande BK, Goupale DC. Development and evaluation of antiinflammatory oleogels of Bosewellia Serata (GUGUL) and Curcuma longa (TURMERIC). Indian Drugs. 2001;38(12):613-6.

25. Sandhya S, Chandrasekhar J, Vinod KR, et al. Preclinical studies of a novel polyherbal phyto-complex hair growth promoting cream. Asian Pac J Trop Biomed. 2012;2:S296-304.

26. Raknam P. Skin evaluation of creams containing Phyllanthus emblica fruit extract liposomes. Thesis Prince of Songkla University, 2012.

27. Ravikumar P, Mallya R. Development and evaluation of a polyherbal topical formulation. JPR: BioMedRx: Int J. 2013;1(7):637-640.

28. Lee KK, Kim JH, Cho JJ, et al. Inhibitory effects of 150 plant extracts on elastase activity, and their anti-inflammatory effects. Int J Cosmet Sci. 1999;21:71-82.

29. Zhao HF, Fan W, Dong JJ, et al. Evaluation of antioxidant activities and total phenolic contents of typical malting barley varieties. Food Chem. 2008;107:296-304.

30. Khan RA, Khan MR, Sahreen S, et al. Evaluation of phenolic contents and antioxidant activity of various solvent extracts of Sonchus asper (L.) Hill. Chem Cent J. 2012;6:12.

31. Bombardelli E, Patri GF. Complex compounds of bioflavonoids with phospholipids: their preparation and use and pharmaceutical and cosmetic compositions containing them, United States patents, patent no 5,043,323, 1991.

32. Silymarin Phytosome ${ }^{\circledR}$ — proven efficacy on humans. Free radical scavenging activity. Mechanism of action for aging and environmental skin protection, http://www.indena.com/pdf/ silymarin_phytosome.pdf (Date accessed 23 Sept 2014).

33. Bombardelli E, Cristoni A, Morazzoni P. Phytosome® in functional cosmetic. Fitoterapia. 1995;LXV(5):387-401.

34. Dey S, Mundkinajeddu D, Manjunath S. Bioactive caffeic acid esters from Glycyrrhiza glabra. Nat Prod Res. 2009;23(18):1657-63. 